This is the final peer-reviewed accepted manuscript of:

S. V. Suraci, D. Fabiani and J. Cohen, "In situ defect recognition analysis on long cables through nondestructive reflectometry and dielectric spectroscopy methods: a comparison," 2020 IEEE Electrical Insulation Conference (EIC), Knoxville, TN, USA, 2020, pp. 41-44

The final published version is available online at:

10.1109/EIC47619.2020.9158583

Rights / License:

The terms and conditions for the reuse of this version of the manuscript are specified in the publishing policy. For all terms of use and more information see the publisher's website.

This item was downloaded from IRIS Università di Bologna (https://cris.unibo.it/)

When citing, please refer to the published version. 


\section{In situ defect recognition analysis on long cables through nondestructive reflectometry and dielectric spectroscopy methods: a comparison}

\author{
Simone Vincenzo Suraci \\ LIMES (Laboratory of Innovative \\ Materials for Electrical Systems) - DEI \\ University of Bologna \\ Bologna, Italy \\ simone.suraci@unibo.it
}

\author{
Davide Fabiani \\ LIMES (Laboratory of Innovative \\ Materials for Electrical Systems) - DEI \\ University of Bologna \\ Bologna, Italy \\ davide.fabiani@unibo.it
}

\author{
Josy Cohen \\ Institut LIST, CEA, Paris-Saclay \\ Campus \\ Nano-INNOV, Bât. 862, \\ 91191 Gif sur Yvette, France. \\ josy.cohen@cea.fr
}

\begin{abstract}
In this paper the aging through high temperature of 10-meter long coaxial cables and its change in electrical properties have been investigated through non-destructive electrical techniques i.e. dielectric spectroscopy and time domain reflectometry. Both techniques allow changes of electrical properties to be revealed with aging, however, the coupling of these two techniques permits an effective cable aging assessment allowing also the recognition of local defects. Indeed, it has been demonstrated that dielectric spectroscopy is more sensitive when the cable is globally aged, while time domain reflectometry, in addition to a global investigation, can also single out aging occurring in limited portion of cable insulation (local aging).
\end{abstract}

Keywords- Time domain reflectometry, dielectric spectroscopy, aging, onsite measurements, cable diagnostics, lv cables.

\section{INTRODUCTION}

Low voltage cables are widely used in nuclear power plants (NPPs) for power transmission, control of equipment and instrumentation and communication of signals and data $[1,2]$. It has been estimated that each NPP owns about 1500 $\mathrm{km}$ of LV cables. Since most of the NPPs built during the ' $80 \mathrm{~s}$ and 90 s are now reaching their end-of-life point, electric utility companies are trying to extend the NPPs operating life up to other 40 years. To do so, $\mathrm{LV}$ cables require a cable aging management system which includes condition monitoring in order to guarantee the cable performance under normal operation as well as accident conditions.

This research is part of a H2020 EU Project called "TeaM Cables" which aims at providing NPP operators a novel methodology for efficient and reliable NPP cable ageing management by developing cable aging models and methodologies for non-destructive testing techniques.

A major requirement for condition monitoring techniques is being non-destructive and applicable on-site. Among the most promising techniques for cable online monitoring, electrical measurements such as dielectric spectroscopy (DS) and time domain reflectometry (TDR) have gained more and more interest in recent years.

The former could provide the evaluation of the trend of the complex permittivity as a function of frequency. Various works showed that the increase of the dielectric losses is directly linked with the degradation state of the cable [3-7]. On the other hand, the TDR technique is used to identify and localize defects in cables detecting discontinuities in the electrical impedance that occur due to inhomogeneities or local defects along the cable length $[2,8,9,10,11]$.

This paper aims at, on the one hand, evaluating the suitability of these two techniques in the study of the evolution and degradation with aging of long cable dielectric properties; on the other hand, at making a comparison between the two techniques in terms of applicability for onsite applications.

The cables analyzed in this work are XLPE cables which have been thermally aged. Aging has been performed, in one case, on the entire cable, in the second case, on a small part in order to simulate a local aging condition. The two aforementioned measurement techniques have been used to characterize the electrical behavior of cable insulation.

\section{MATERIALS AND METHODS}

\section{A. Cable specimens}

The samples here analyzed are low-voltage I\&C coaxial cables with XLPE insulation used in NPPs, especially designed for the project. Morphology of the investigated cables is reported in Fig. 1. Specimens are made of five concentric parts:

1. Conductor-Copper (the innermost);

2. Primary insulation - XLPE;

3. PET Polymeric film;

4. Shielding - Copper wire braid;

5. Outer sheath - Low Smoke Zero Halogen.

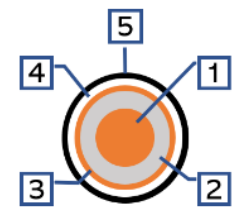

Fig. 1. Multilayer structure of coaxial cables under investigation. (1) Conductor - Copper, (2) Primary insulation - XLPE, (3) Polymeric film PET, (4) Shielding - Copper wire braid, (5) Sheath - Low smoke zero halogen 
The primary insulation, in particular, is a silane crosslinked polyethylene stabilized with 1 phr of primary antioxidant (phenol-based) and 1 phr of secondary antioxidant (thioetherbased). Each cable specimen is about $10 \mathrm{~m}$ long.

\section{B. Cables aging}

Aging has been performed through high temperature in oven at $150^{\circ} \mathrm{C}$ for three weeks. In order to investigate the ability of TDR to localize aged spots through the entire cable system, aging has been performed on one cable throughout the entire cable length and on a second cable only locally following the scheme reported in Figure 2.

\begin{tabular}{|c|c|c|c|c|c|}
\hline $196 \mathrm{~cm}$ & $124 \mathrm{~cm}$ & $200 \mathrm{~cm}$ & $99 \mathrm{~cm}$ & $200 \mathrm{~cm}$ & $75 \mathrm{~cm}$ \\
\hline & 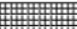 & & 無蟱册 & & 帚册册 \\
\hline
\end{tabular}

Fig. 2. Scheme and aged part lenghts for local aging cable. Grey parts are the ones subjected to aging inside the oven, white parts unaged.

\section{Dielectric spectroscopy measurements}

Capacitance and $\tan \delta$ have been investigated through the setup showed in Figure 3. This is made up of:

1. a function signal generator (Rigol DG 1022z) which applies voltage equal to $4 \mathrm{~V}_{\text {rms }}$ with frequency in the range $1-200 \mathrm{kHz}$;

2. a device mainframe made up of a data acquisition module consisting of NI cDAQ9174 model and NI9215 data acquisition card, two sampling resistor modules, four BNC input channels for the signal measurement and one input channel for the signal source;

3. A laptop to acquire data.

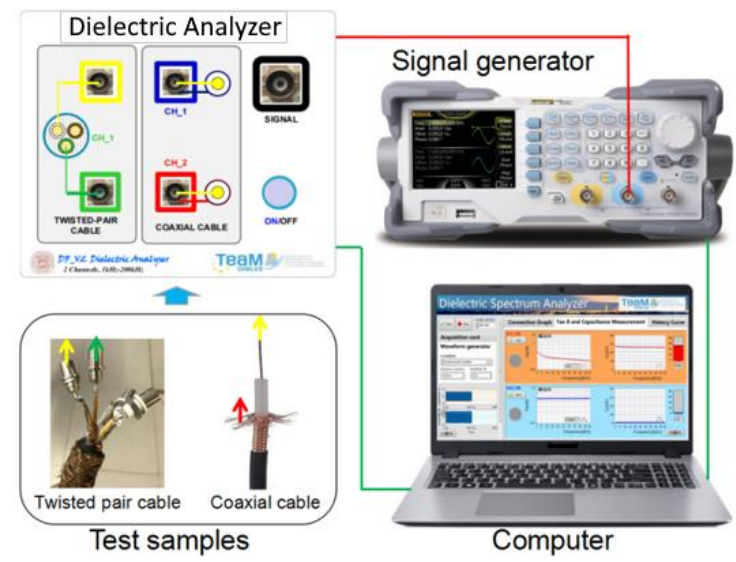

Fig. 3. Measurements setup scheme for dielectric spectroscopy tests

\section{Time domain reflectometry (TDR) measurements}

A ZNB8 Vector Network Analyzer (VNA) from Rodhe\&Schwarz has been used to perform TDR measurements. The VNA setup (Figure 4) has been calibrated with VNA reference impedance $\mathrm{Z}_{0}=50 \mathrm{Ohms}$. The frequency region analyzed is between $50 \mathrm{kHz}$ and 1.000050 GHz. Data output is made up of 20001 points at $0 \mathrm{dBm}$ power. The frequency band and the number of point has been chosen to obtain a hamornic grid.

This setup gives the reflection coefficient $(\rho)$, defined as:

$$
\rho=\frac{Z c-Z_{0}}{Z c+Z_{0}}
$$

with $Z_{c}=$ characteristic impedance of cable under test $\mathrm{Z}_{0}=$ the calibration impedance of the VNA

Then, to obtain the characteristic impedance of the cable under test $\mathrm{Z}_{\mathrm{c}}$ :

$$
Z c=Z_{0} \frac{1+\rho}{1-\rho}
$$

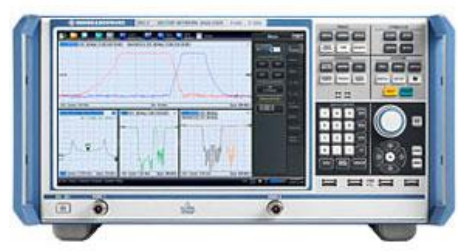

Fig. 4. ZNB8 Vector Network Analyzer used for TDR measurements

\section{RESULTS}

\section{A. Dielectric spectroscopy results}

Figure 5 displays the results obtained through the dielectric spectroscopy setup. Figure 5.a and 5.b shows the trend for the three cables considered of Capacitance and $\tan \delta$, respectively.

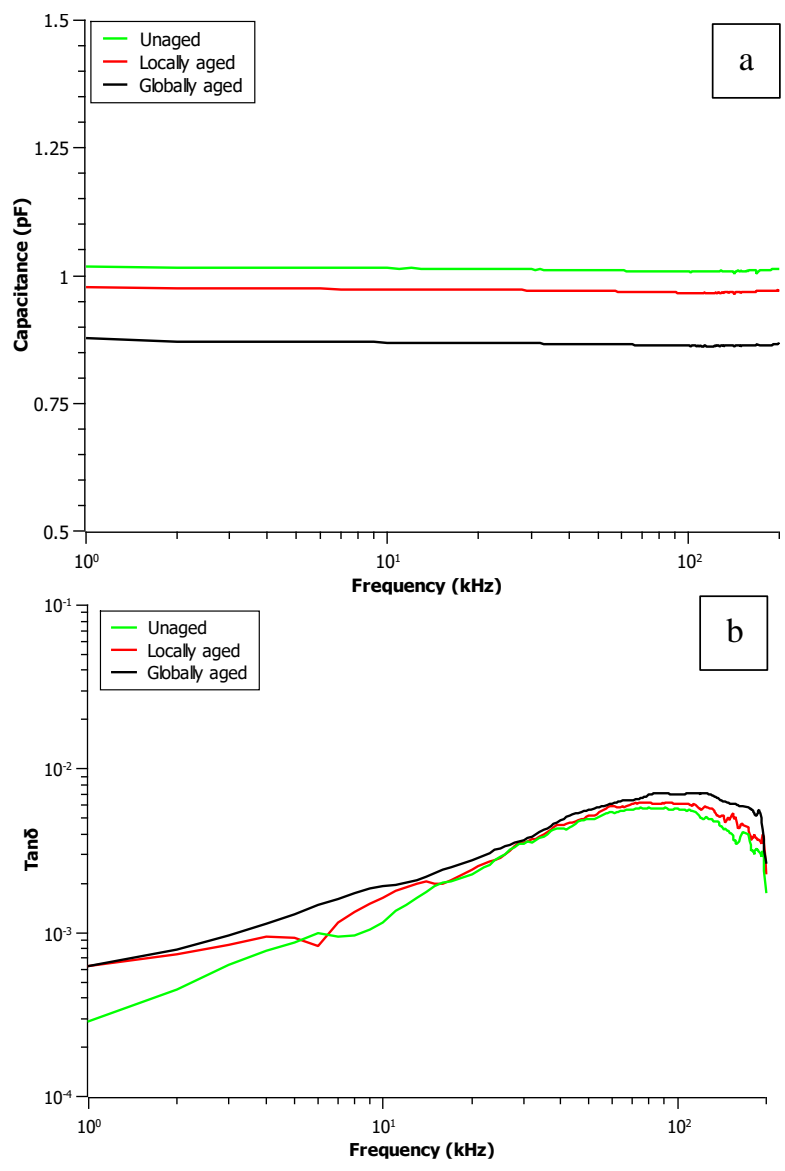

Fig. 5. Dielectric spectroscopy results for differently aged cables (a) Capacitance (b) $\tan \delta$

As showed (Figure 5.a), capacitance values decrease by about $15 \%$ for the global aged sample with respect to the unaged one. This behavior, quite unusual, could be imputable to the very high temperature set during aging. As a matter of fact, this can cause a detachment of the external metallic screen bringing to a slight reduction of the analysis area and consequently a reduction of the resulting capacitance values. In the case of the local aged cable, the reduction is almost 
negligible (by $0.04 \mathrm{pF}$ ) and it could be imputable to measurements setup uncertainties.

Focusing of the dissipation factor trend (Figure 5.b), one can state that, as expected, the $\tan \delta$ increases with the increase of aging severity. Indeed, since the dielectric measurement is a bulk measurement which takes into account the whole equipment under test (EUT), the local aged spots result in a little global increase of the $\tan \delta$ value throughout the frequency region here analyzed. Fixing a frequency i.e. $100 \mathrm{kHz}$, it is possible to claim that this increase imputable to the local aging condition is anyway lower (of about 2.5 times) than the one referred to the global aging.

\section{B. Time domain reflectometry $(T D R)$ results}

For the new coaxial sample (Figure 6), it is possible to highlight some small noise between the first peak and the last peak representing little heterogeneities throughout the cable length signal. Measurements showed no important modifications of the characteristic impedance $\left(\mathrm{Z}_{\mathrm{c}}\right)$. To evaluate $Z_{c}$ equation (2) is used leading to $Z_{c}=55.2$ Ohms.

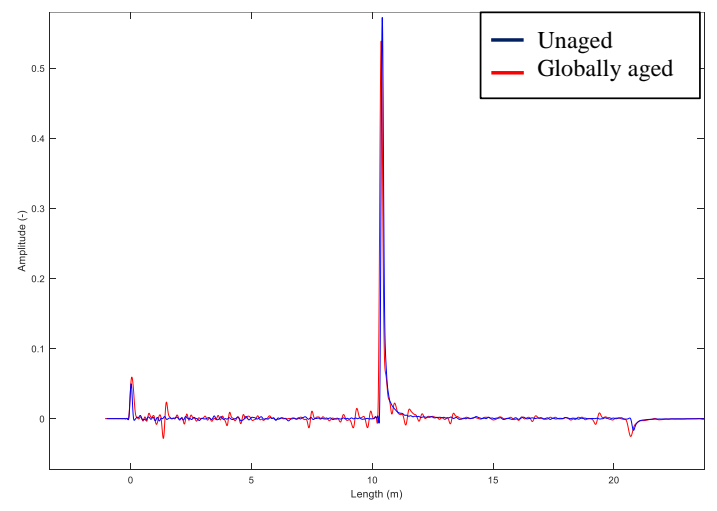

Fig. 6. TDR measurement of the new coaxial and globally aged sample

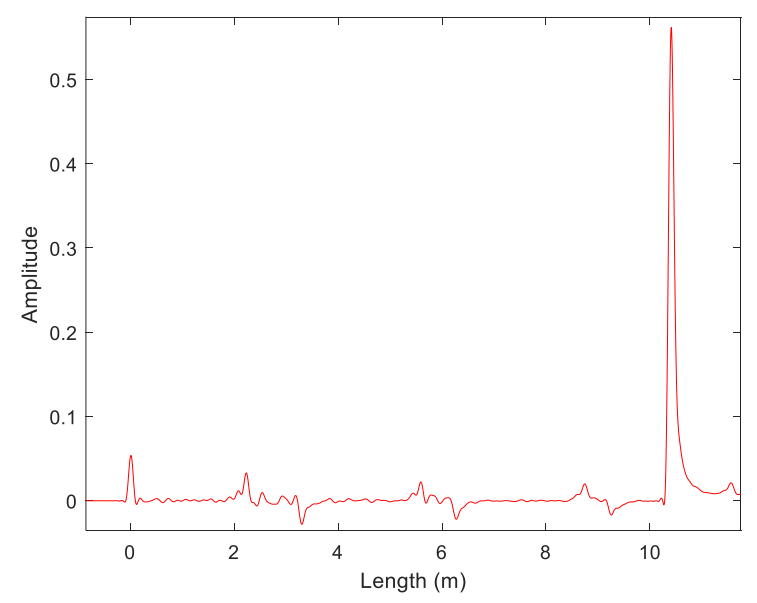

Fig. 7. TDR measurement of the locally aged coaxial sample

For the globally aged coaxial sample (Figure 6), some heterogeneities, evenly distributed along the cable length, are visible between the first peak and the last bigger peak of open circuit. Using the same equation with the first peak (2), $\mathrm{Z}_{\mathrm{c}}=$ 56,3 Ohms.
Focusing on the locally aged coaxial sample, it is possible to underline some heterogeneities between the first peak and the last peak of open circuit located at $10.41 \mathrm{~m}$ (Figure 7) placed in three defined areas. These local heterogeneities result to be placed at the same position and to have the same length $(1.25$ $\mathrm{m}, 1 \mathrm{~m}$ and $0.75 \mathrm{~m}$ ) of the local aged segments (Figure 2). To evaluate the characteristic impedance $Z_{c}$ we use equation (2) in recursive way to obtain Figure 9.

In this case, the impedance increases by about $10 \%$ in the segments where the cable has been locally aged.

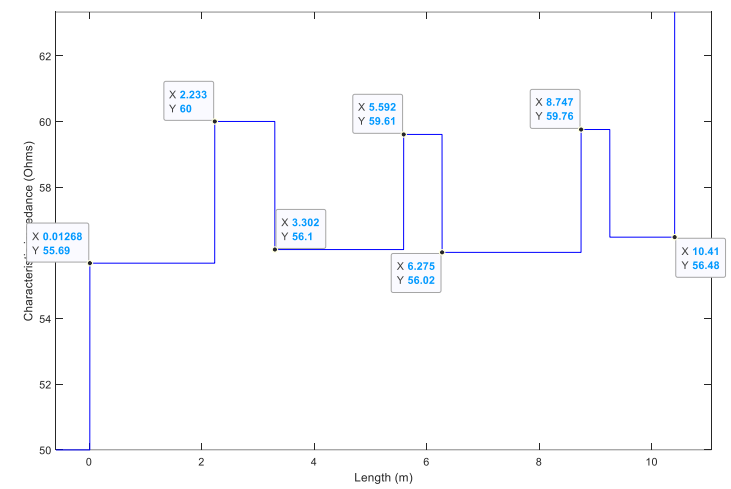

Fig. 8. $\mathrm{Z}_{\mathrm{c}}$ profile for the locally aged coaxial sample

\section{DISCUSSION}

In the previous section it has been shown that different aging conditions lead to different behavior of the electrical quantities analyzed here. In particular, a good consistency between the two electrical measurements techniques proposed (TDR and dielectric spectroscopy) is evidenced.

The dielectric spectroscopy measurements, especially the dissipation factor $(\tan \delta)$, showed in literature [3-7] to be easily relatable to cable insulation health and status. Broadening this concept, the evolution of $\tan \delta$ with aging can be related to the degradation of the insulation material. In particular, the frequencies nearby $100 \mathrm{kHz}$ have been [4-7] used as an aging marker since they resulted to be associated with polymer oxidation, as shown by correlation with the most common state-of-the-art aging assessment techniques, which are usually destructive (e.g. tensile stresses, thermo-chemical analyses) [4, 7]. This type of testing, however, is a bulk analysis because the quantities obtained like e.g. $\tan \delta$, are not affected significantly by local defects or damages, but depends on the insulation properties of the entire cable system.

On the other side, the time domain reflectometry (TDR) measures reflections of an impulse signal along a single conductor to detect and locate any changes in the cable impedance. If the cable owns a homogenous impedance, there will be no reflection between the initial peak and the final peak (i.e. open circuit). On the contrary, if the cable shows any variation of impedance, part of the initial signal will be reflected back to the source, resulting in little peaks (as showed in Figures 6-8). The reflected signal can be linked to distance along the cable and its peak amplitude allows the estimation of the impedance change. It is evident that TDR tests are capable to assess the condition of the entire cable, but very little information are referred to changes in the insulation $[9,2,12]$. 
Results from the TDR measurements report that if the aging is globally displaced, the increase of impedance is very little (about $1 \mathrm{Ohm}$ ) considering the first peak, while if the aging is placed locally, the local increase of impedance is way higher (about 5 Ohms), and located in correspondence of the aged segments.

On the contrary, the variation of the other two dielectric properties (Cap and $\tan \delta$ ) is almost negligible in the case of local aging with respect to the unaged cable. Indeed, as already explained, the dielectric spectroscopy is a bulk measurement and it is not able to characterize and localize local dielectric properties. The effect produced results in a variation of the dielectric properties similar to a cable subjected to a very low, but homogenously distributed, aging.

The global aging gives a raise to the dielectric losses which result to be about 2.5 times higher than the unaged material, as stated above. The uniformity of the aging is also globally confirmed by the TDR measurements (Figure 6), in which small peaks, representing the reflection of the signal, which are usually related to the contact of the conductor with aged insulation, are evenly distributed throughout the cable length.

It has to be pointed out that the actual aging time and setup, particularly on for the local aged cable, did not provide a large modification of the electrical quantities investigated.

However, it is worth commenting that, even if the aging is far away from bringing to the actual cable crisis, the two measurements techniques showed to be sensitive also to these low stressing conditions, confirming the ability of these systems to follow the aging of the cable systems.

This approach opens new opportunities for cable monitoring online. Indeed, the possibility to couple a portable dielectric spectroscopy measurement device together with a TDR-capable VNA can permit to easily assess in depth the health of the cable system, in a non-destructive way. In fact, an initial evaluation through the dielectric spectroscopy setup allows the definition of the global status of the cable insulation matter and its capability to fulfill its role without any damage to the system. A secondary test through TDR setup, permits the evaluation and identification of local aging defects and cable weak points. It is worth commenting that, anyway, global aging can be evaluated also through TDR with a different measurement protocol $[9,10,11,12]$.

\section{CONCLUSIONS}

This article introduced an innovative way for the assessment of the status of cable systems on-site in a nondestructive manner. The two presented measurements techniques: the dielectric spectroscopy and the time domain reflectometry, showed to be consistent with each other and the possibility to combine them allows the assessing the global health and localize any possible defects inside the analyzed cable system. This approach would be a key step forward for the state-of-the-art cable condition monitoring online. In this way power plants management companies could easily access to the cable system status and, in case, schedule in advance the replacement of the entire or part of the cable system.

Future works on this topic will focus on the applicability of these techniques to other morphologies of cables which are present inside the most common power plants i.e. twisted pair cables, and to more severely aged cable systems.

\section{ACKNOWLEDGMENTS}

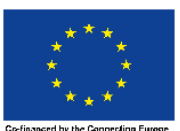

The project leading to this application has received funding from the Euratom research and training programme 2014-2018 under grant agreement No 755183. The authors are grateful to Nexans and UJV for sample preparation and aging.

\section{REFERENCES}

[1] A. Sriraman, N. Bowler, S. W. Glass, and L. S. Fifield, "Dielectric and Mechanical Behavior of Thermally Aged EPR/CPE Cable Materials," in 2018 IEEE Conference on Electrical Insulation and Dielectric Phenomena (CEIDP), 2018, pp. 598-601, doi: 10.1109/CEIDP.2018.8544855.

[2] S. W. Glass, L. S. Fifield, G. Dib, J. R. Tedeschi, A. M. Jones, and T. S. Hartman, "State of the Art Assessment of NDE Techniques for Aging Cable Management in Nuclear Power Plants FY2015," PNNL-24649, M2LW--15OR0404024, 1242348, Sep. 2015.

[3] A. Sriraman, N. Bowler, S. W. Glass, and L. S. Fifield, "Dielectric and Mechanical Behavior of Thermally Aged EPR/CPE Cable Materials," in 2018 IEEE Conference on Electrical Insulation and Dielectric Phenomena (CEIDP), 2018, pp. 598-601, doi: 10.1109/CEIDP.2018.8544855.

[4] S. V. Suraci, D. Fabiani, A. Xu, S. Roland, and X. Colin, "Ageing Assessment of XLPE LV Cables for Nuclear Applications Through Physico-Chemical and Electrical Measurements," IEEE Access, vol. 8, pp. 27086-27096, 2020, doi: 10.1109/ACCESS.2020.2970833.

[5] S. V. Suraci, D. Fabiani, S. Bulzaga, and L. Mazzocchetti, "Investigation of Thermal Degradation of LDPE-Based Materials Through Electrical Measurements," in 2018 IEEE 2nd International Conference on Dielectrics (ICD), 2018, pp. 1-4, doi: 10.1109/ICD.2018.8514643.

[6] S. V. Suraci, D. Fabiani, L. Mazzocchetti, V. Maceratesi, and S. Merighi, "Investigation on Thermal Degradation Phenomena on Low Density Polyethylene (LDPE) through Dielectric Spectroscopy," in 2018 IEEE Conference on Electrical Insulation and Dielectric Phenomena (CEIDP), 2018, pp. 434-437, doi: 10.1109/CEIDP.2018.8544734.

[7] S. V. Suraci, D. Fabiani, L. Mazzocchetti, and L. Giorgini, "Degradation Assessment of Polyethylene-Based Material Through Electrical and Chemical-Physical Analyses," Energies, vol. 13, no. 3, p. 650, Jan. 2020, doi: 10.3390/en13030650.

[8] S. W. Glass, A. M. Jones, L. S. Fifield, and T. S. Hartman, "Frequency domain reflectometry NDE for aging cables in nuclear power plants," presented at the 43RD ANNUAL REVIEW OF PROGRESS IN QUANTITATIVE NONDESTRUCTIVE EVALUATION, VOLUME 36, Atlanta, Georgia, USA, 2017, p. 080015, doi: 10.1063/1.4974640.

[9] S. Sallem and N. Ravot, "Self-adaptive correlation method for soft defect detection in cable by reflectometry," in IEEE SENSORS 2014 Proceedings, Valencia, Spain, 2014, pp. 2114-2117, doi: 10.1109/ICSENS.2014.6985455.

[10] W. Ben Hassen, M. Gallego Roman, B. Charnier, N. Ravot, A. Dupret, A. Zanchetta, F. Morel, "Embedded OMTDR Sensor for Small Soft Fault Location on Aging Aircraft Wiring Systems", Procedia Engineering, vol. 168, pp. 1698-1701, 2016.

[11] A. Lelong, M. Olivas, "On line wire diagnosis using multicarrier time domain reflectometry for fault location", IEEE Sensors Conference, pp. 751-754, October 2009.

[12] M. Franchet, N. Ravot, N. Grégis, J. Cohen, O. Picon, "New Advances in Monitoring the Aging of Electric Cables in Nuclear Power Plants", Advanced Electromagnetics Symposium AES 2012, Paris, 2012 